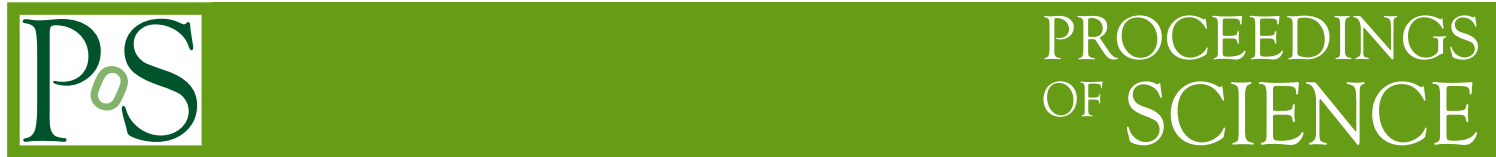

\title{
Lattice QCD and Kaon Physics
}

\author{
Jack Laiho* \\ Department of Physics, Syracuse University, Syracuse, NY 13244 \\ E-mail: jwlaihodsyr.edu
}

I review Lattice QCD with an emphasis on kaon physics. I give a brief introduction to lattice methods and present an overview of the calculations of light quark masses, kaon and pion decay constants, semileptonic kaon decays, and kaon mixing. I also discuss the recent progress made in calculating $K \rightarrow \pi \pi$ matrix elements.

2013 Kaon Physics International Conference,

29 April-1 May 2013

University of Michigan, Ann Arbor, Michigan - USA

\footnotetext{
*Speaker.
} 


\section{Introduction to Lattice QCD}

Kaon physics has historically been the source of many important discoveries that have shaped our understanding of particle physics, and it is possible that more may be in store. A number of precision measurements in kaon physics are available that further constrain physics beyond the Standard Model, but the reach of these constraints could be improved if the theory errors were reduced. Most of these uncertainties are due to hadronic physics that is difficult to calculate due to the nonperturbative nature of QCD at long distance scales. These uncertainties can be reduced by further improvements to the primary nonperturbative method used to evaluate the effects of the strong interactions on weak decays, namely Lattice QCD. Lattice QCD is a nonperturbative definition of QCD that allows for a direct numerical evaluation of the Feynman path integral that defines the field theory.

In Lattice QCD, Euclidean space-time is discretized, and the continuum limit is reached by tuning the bare coupling to zero. The only parameters that enter the theory are fundamental parameters of the Standard Model: the quark masses and the strong coupling constant. When weak interactions are included, one makes use of the operator product expansion to separate low and high energy scales. Long-distance weak matrix elements are then multiplied by Wilson coefficients that encode the short distance physics where heavier scales (e.g. the $W$ and $Z$ boson masses) have been integrated out; these coefficients can be calculated perturbatively. A Standard Model prediction for a typical observable then takes the form

$$
\Gamma=(\text { known factor })(\mathrm{CKM} \text { factor)(non-perturbative } \mathrm{QCD} \text { factor), }
$$

where the known factor is a combination of kinematic factors and well-known constants, the CKM factor comes from the CKM quark mixing matrix that determines the amplitude for flavor changing between quarks, and the QCD factor parameterizes the nonperturbative hadronic physics.

The continuum path integral that defines QCD is

$$
\langle\mathscr{O}\rangle=\frac{1}{Z} \int D \mathscr{U} D \psi_{\text {sea }} D \bar{\psi}_{\text {sea }} e^{-S_{\mathrm{QCD}}\left[\mathscr{U}, \psi_{\text {sea }}, \bar{\psi}_{\text {sea }}\right]} \mathscr{O}\left[\mathscr{U}, \psi_{\text {val }}, \bar{\psi}_{\text {val }}\right],
$$

where $S_{\mathrm{QCD}}$ is the Euclidean QCD action, $\mathscr{U}$ represents the gauge fields, $\psi_{\text {sea }}\left(\bar{\psi}_{\text {sea }}\right)$ is the sea valence quark (anti-quark) field, and $\psi_{\text {val }}\left(\bar{\psi}_{\text {val }}\right)$ is the valence quark (anti-quark) field. The operator $\mathscr{O}$ is the operator whose expectation value is being taken, and $Z$ is the partition function that normalizes the operator expectation value so that $\langle 1\rangle=1$. Lattice calculations reduce this path integral to a multi-dimensional integral. The integral is still very large, with dimension equal to $N_{s}^{3} \times N_{t} \times 12 \times N_{f}$, where $N_{s}$ and $N_{t}$ are the length of the lattice in the space and time directions, respectively, $N_{f}$ is the number of flavors of quarks, and 12 is the number of colors times the number of spinor degrees of freedom.

The only practical way to evaluate such a large integral is through Monte Carlo importance sampling. The errors that one encounters in interpreting lattice calculations are a result of the approximations made when evaluating this integral. The finite box-size leads to finite volume errors, the finite lattice spacing leads to continuum extrapolation errors. It is expensive to simulate with the $u$ and $d$ quarks at their physical values. Usually, calculations are done for heavier values of these quark masses and an extrapolation is made to the physical values, often with the help of chiral 

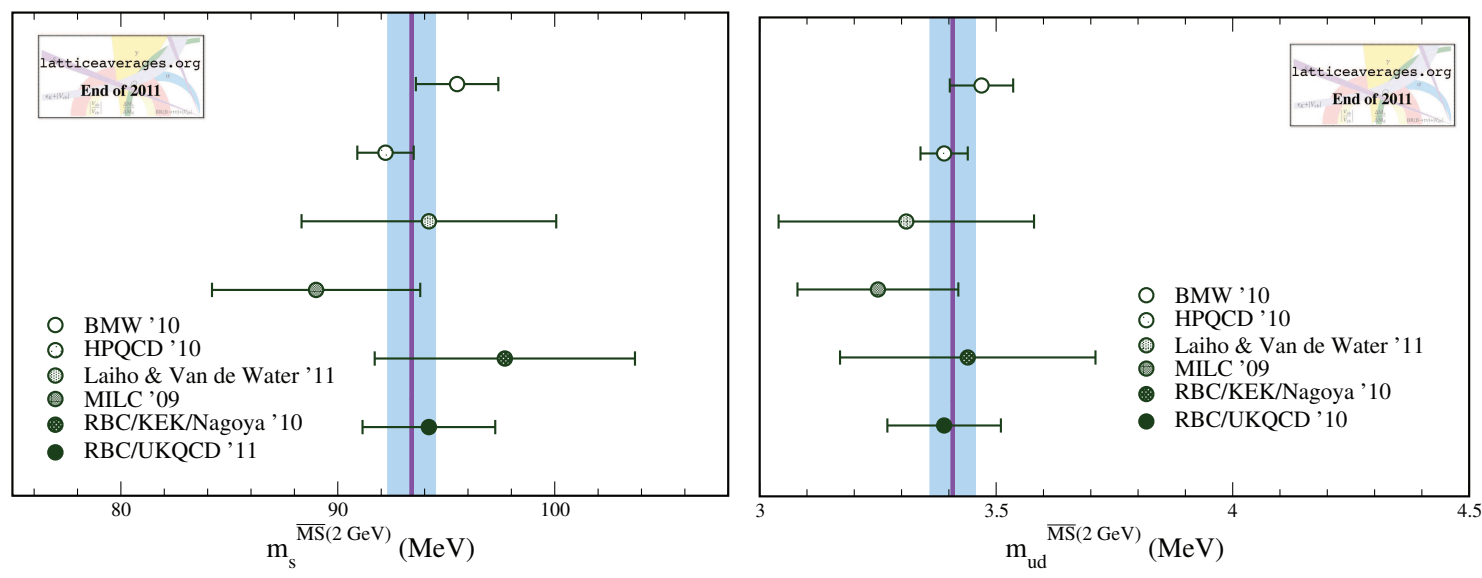

Figure 1: Left panel: The strange quark mass $m_{s}^{\overline{M S}}(2 \mathrm{GeV})$ from many groups, including the world average [6]. Right panel: The results for the average of the $u$ and $d$ quark masses $m_{u d}^{\overline{M S}}(2 \mathrm{GeV})$ from many groups with world average. Results in both figures are taken from Refs. [3, 4, 7, 8, 10,11].

perturbation theory. Thus, many calculations also have a chiral extrapolation error [1], though some groups now have results from calculations done directly at physical light quark masses. Another error appears for scheme-dependent quantities like quark masses, where a conversion must be made from the lattice regulator scheme to a continuum scheme like $\overline{M S}$. Such conversions are typically done using non-perturbative methods that make contact with a perturbative expansion, ideally at a high scale where perturbation theory works well. Such matching factors come with their own systematic errors, including a perturbative truncation error.

For a more in depth review of Lattice QCD and additional references, see Ref. [2].

\section{Quark masses}

The quark masses are fundamental parameters of the Standard Model, but due to confinement the quarks do not appear as free particles in nature. One must tune the lattice quark masses so that one reproduces the experimentally known hadron spectrum, with three experimental inputs needed to determine the three light quark masses and a fourth experimental input to fix the scale. An additional input is also needed if the charm quark is included in the calculation. The bare lattice quark masses are then known in the regularization scheme defined by the lattice action of any particular calculation, but to compare to results using other lattice actions or to be used as input in continuum calculations, must be converted to a standard continuum regularization scheme like $\overline{M S}$. This matching is the most difficult part of the calculation, and it is only in the last few years that it has been brought under control by different groups using various improved nonperturbative methods (see e.g., Refs. [3, 4, 5]).

Recent results for the strange quark mass and for the averaged up and down quark mass $m_{u d}$ are shown in Fig. 1. The agreement between the different results for quark masses is striking, especially given the long history of disagreement between various $m_{s}$ determinations ${ }^{1}$.

\footnotetext{
${ }^{1}$ This can be seen in the FLAG lattice review [12], which shows the history of determinations of $m_{s}$.
} 


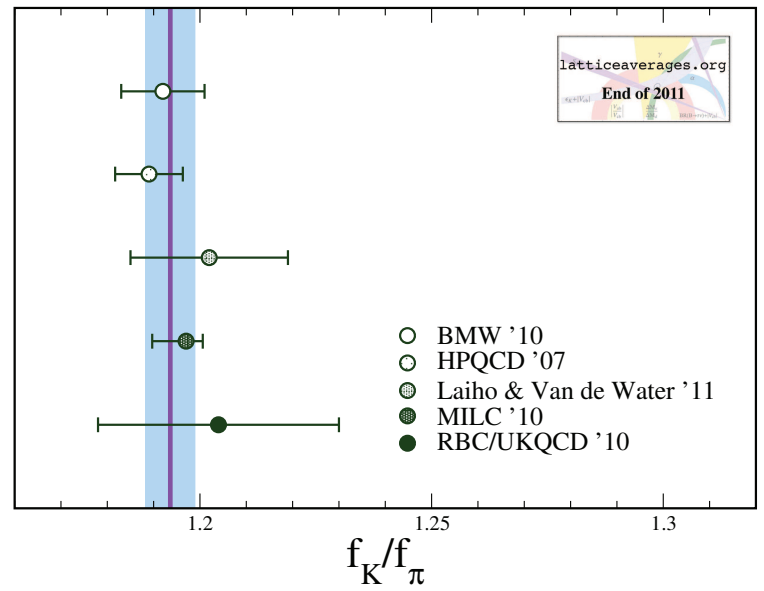

Figure 2: Results for $f_{K} / f_{\pi}$. The results are quoted from Refs. [10, 14, 15, 16, 17].

\section{Decay constants}

The light pseudoscalar decay constants $f_{K}$ and $f_{\pi}$ are useful benchmarks for lattice calculations, and their ratio is interesting because it allows for the determination of the ratio of CKM matrix elements $\left|V_{u s}\right| /\left|V_{u d}\right|$ from kaon and pion leptonic branching fractions,

$$
\frac{\Gamma\left(K \rightarrow \ell \bar{v}_{\ell}\right)}{\Gamma\left(\pi \rightarrow \ell \bar{v}_{\ell}\right)}=\left(\frac{\left|V_{u s}\right|}{\left|V_{u d}\right|}\right)^{2}\left(\frac{f_{K}}{f_{\pi}}\right)^{2} \frac{m_{K}\left(1-\frac{m_{\ell}^{2}}{m_{K}^{2}}\right)^{2}}{m_{\pi}\left(1-\frac{m_{\ell}^{2}}{m_{\pi}^{2}}\right)^{2}}\left[1+\frac{\alpha}{\pi}\left(C_{K}-C_{\pi}\right)\right],
$$

where $m_{i}$ is the mass of a lepton or meson, $\alpha$ is the QED coupling, and $C_{K}$ and $C_{\pi}$ are perturbative factors encoding short distance electroweak corrections [13].

A relatively recent average of $f_{K} / f_{\pi}$ is shown in Fig. 2. Since this average was done there have been two additional precision lattice determinations of $f_{K} / f_{\pi}$. One is from the MILC Collaboration, who quote $f_{K} / f_{\pi}=1.1947(26)(37)$, where the first error is statistical and the second is the total systematic [18]. The second is from HPQCD, who quote 1.1916(21) [19]. For more details see Ref. [20].

\section{4. $K \rightarrow \pi \ell v$ semileptonic decay}

The semileptonic $K \rightarrow \pi \ell v$ decay can be used to obtain the CKM matrix element $V_{u s}$ from the experimental branching fraction using [21]

$$
\Gamma_{K \ell 3}=\frac{G_{F}^{2} m_{K}^{5}}{192 \pi^{2}} C_{K}^{2} S_{E W}\left(\left|V_{u s}\right| f_{+}\right)^{2} I_{K \ell}\left(1+\delta_{E M}^{K \ell}+\delta_{S U(2)}^{K \pi}\right)^{2},
$$

where $S_{E W}=1.0232(3)$ is the short-distance electroweak correction, $C_{K}$ is a Clebsch-Gordan coefficeint, $f_{+}(0)$ is the form factor at zero momentum transfer, and $I_{K \ell}$ is a phase-space integral that is sensitive to the momentum dependence of the form factors. The quantities $\delta_{E M}^{K \ell}$ and $\delta_{S U(2)}^{K \pi}$ are long-distance EM corrections and isospin corrections, respectively. The value

$$
\left|V_{u s}\right| f_{+}(0)=0.2163(5)
$$




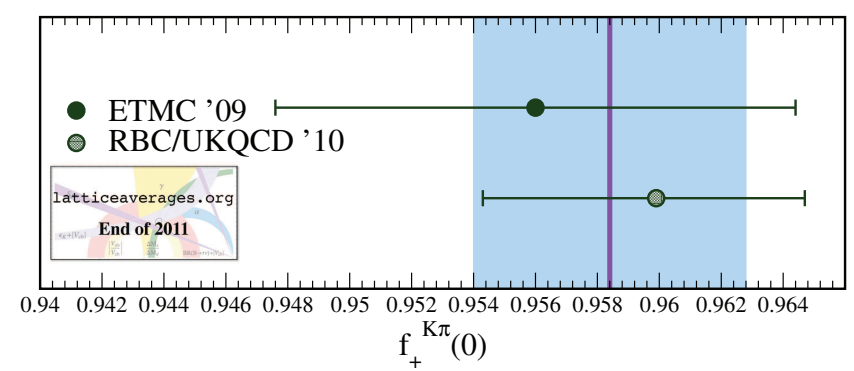

Figure 3: Results for $f_{+}(0)$. The results are quoted from Refs. [25, 26].

has been determined from experimental measurements of $K \rightarrow \pi \ell v$ decays and non-lattice theory for the other inputs to Eq. (4.1) [21]. The non-perturbative information is encoded in the form factor $f_{+}(0)$, and once this is known from lattice $\mathrm{QCD}$, a value for $V_{u s}$ can be determined. The value of $f_{+}(0)$ is already rather well constrained by $S U(3)$ chiral perturbation theory, an expansion in powers of $m_{K}^{2} /\left(8 \pi^{2} f_{\pi}^{2}\right)$ [22]. One can write $f_{+}(0)=1+f_{2}+f_{4}+\ldots$, where the first term is equal to one due to current conservation in the $S U(3)$ limit. The correction $f_{2}$ does not contain any new unknown low energy constants, as required by the Ademollo-Gatto theorem [23], and is predicted in terms of pion and kaon masses and the pion decay constant to be $f_{2}=-0.0226$. One needs to know $f_{4}$, if one is to do better, but this requires the determination of new higher order unknown low energy constants. The value for $f_{4}$ was estimated by Leutwyler and Roos in 1984 [24] using a quark model; they obtained $f_{4}=-0.016(8)$, which gives $f_{+}(0)=0.961(8)$.

An average of results for $f_{+}(0)$ with full systematic error budgets as of 2011 is shown in Fig. 3. In addition to these results by the RBC/UKQCD and ETM Collaborations [25, 26], there is a more recent published result from FNAL/MILC, where the value $f_{+}(0)=0.9667(23)(33)$ is quoted [27]. The first error is statistical and the second is the total systematic error. There is also a preliminary result from JLQCD, where they quote $f_{+}(0)=0.959(6)(5)$, and the errors are statistical and systematic [28]. RBC/UKQCD and FNAL/MILC are working on calculations that improve upon previous results [29, 30]. For more details see Ref. [20].

\section{Kaon mixing}

The constraint on the unitarity triangle coming from kaon mixing can be expressed as

$$
\left|\varepsilon_{K}\right|=C_{\varepsilon} \kappa_{\varepsilon} B_{K} A^{2} \bar{\eta}\left\{-\eta_{1} S_{0}\left(x_{c}\right)\left(1-\lambda^{2} / 2\right)+\eta_{3} S_{0}\left(x_{c}, x_{t}\right)+\eta_{2} S_{0}\left(x_{t}\right) A^{2} \lambda^{2}(1-\bar{\rho})\right\},
$$

where $C_{\varepsilon}$ is a collection of experimentally determined parameters, $\kappa_{\varepsilon}$ represents long-distance contributions and a correction due to the fact that the quantity $\phi_{\varepsilon} \neq 45$ degrees [31], $B_{K}$ is the kaon bag parameter, the $\eta_{i} S_{0}$ are perturbative coefficients, and $\lambda, A, \bar{\rho}, \bar{\eta}$ are CKM matrix elements in Wolfenstein parameterization. The experimental determination of $\left|\varepsilon_{K}\right|$ leads to a constraint on the unitarity triangle in the shape of a hyperbolic band in the $\bar{\rho}-\bar{\eta}$ plane. The main non-perturbative input needed from the lattice to implement this constraint on the CKM unitarity triangle is the kaon bag parameter $B_{K}$. The world average for $\hat{B}_{K}$ is shown in Fig. 4 . All of the results are in good agreement, which is impressive given the different discretizations and methods employed in the various calculations. 


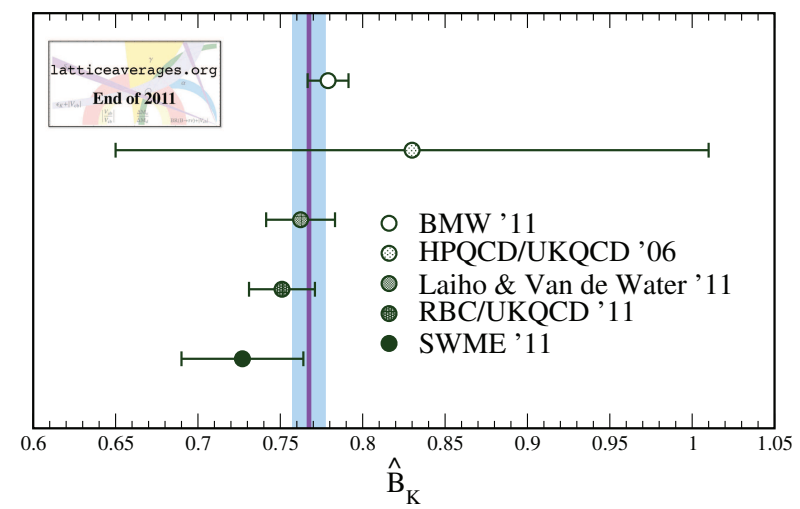

Figure 4: Results for $\hat{B}_{K}$. The results are quoted from Refs. [10, 11, 32, 33, 34].

\section{6. $K \rightarrow \pi \pi$}

Lattice calculations of non-leptonic $K \rightarrow \pi \pi$ decays are challenging because of the two-hadron final state, but they are important for phenomenology. Lattice calculations of $K \rightarrow \pi \pi$ matrix elements have the potential to give us a first principles determination of the decades old $\Delta I=$ $1 / 2$ rule, and would finally allow us to use the experimental measurement of $\varepsilon^{\prime} / \varepsilon$ as a precision constraint on the Standard Model [35]. The Standard Model prediction for $\varepsilon^{\prime} / \varepsilon$ is

$$
\operatorname{Re}\left(\frac{\varepsilon_{K}^{\prime}}{\varepsilon_{K}}\right) \approx \frac{\omega}{\sqrt{2}\left|\varepsilon_{K}\right|}\left[\frac{\operatorname{Im}\left(A_{2}\right)}{\operatorname{Re}\left(A_{2}\right)}-\frac{\operatorname{Im}\left(A_{0}\right)}{\operatorname{Re}\left(A_{0}\right)}\right]
$$

where $A_{0}$ and $A_{2}$ are the amplitudes for $K \rightarrow \pi \pi$ decays into definite isospin states, and real and imaginary refer only to the part of the amplitude that becomes complex due to the presence of the weak phase. The smallness of the parameter $\omega=\operatorname{Re}\left(A_{2}\right) / \operatorname{Re}\left(A_{0}\right) \approx 0.05$ is a manifestation of the $\Delta I=1 / 2$ rule.

$K \rightarrow \pi \pi$ matrix elements are difficult to calculate on the lattice because the Maiani-Testa nogo theorem [36] tells us that we cannot extract physical matrix elements from Euclidean correlation functions with multi-hadronic final states. Due to the restriction of working in Euclidean time, the most straightforward lattice implementation of calculating $K \rightarrow \pi \pi$ matrix elements only works if the final state pions are at rest, or at some other set of unphysical kinematics. Two general strategies have emerged for getting around this problem. One strategy is to construct $K \rightarrow \pi \pi$ matrix elements indirectly using the low energy constants (LEC's) of chiral perturbation theory as determined from simpler lattice matrix elements such as $K \rightarrow 0$ and $K \rightarrow \pi$ [37]. It was shown in Refs. $[38,39,40,41]$ that all LEC's through next-to-leading order could be obtained from relatively simple lattice quantities. However, this method has the disadvantage that the convergence of $S U(3)$ chiral perturbation theory at the physical kaon mass is slow, and it is not clear whether $K \rightarrow \pi \pi$ matrix elements can be computed in this way to a useful precision [42, 43].

A method for calculating $K \rightarrow \pi \pi$ matrix elements directly at physical kinematics was introduced by Lellouch and Lüscher [44]. The Lellouch-Lüscher method exploits the finite lattice volume to obtain the matrix elements directly by tuning the volume so that the first excited state of the two pion state matches the kaon mass. The direct method is straightforward to implement, 
Table 1: Estimated total error budget for $\operatorname{Re}\left(A_{2}\right)$ from RBC/UKQCD [50]. Each source of uncertainty is given as a percentage.

\begin{tabular}{ccc}
\hline \hline uncertainty & $\operatorname{Re}\left(A_{2}\right)$ & $\operatorname{Im}\left(A_{2}\right)$ \\
\hline statistics & $4.3 \%$ & $7.5 \%$ \\
finite lattice spacing & $15 \%$ & $15 \%$ \\
finite volume errors & $6.0 \%$ & $6.5 \%$ \\
Partial quenching effect & $3.5 \%$ & $1.7 \%$ \\
operator renormalization & $1.8 \%$ & $5.6 \%$ \\
unphysical kinematics & $0.4 \%$ & $0.8 \%$ \\
derivative of the phase shift & $0.97 \%$ & $0.97 \%$ \\
Wilson coefficient & $6.6 \%$ & $6.6 \%$ \\
\hline total & $18 \%$ & $19 \%$ \\
\hline \hline
\end{tabular}

though it is computationally demanding because it requires large lattice volumes ( $\sim 6 \mathrm{fm}$ ) and physical light quark masses. Improvements to the method have been introduced so that the non-zero momentum pion state becomes the ground state and smaller volumes can be used [45, 46, 47, 48]. The RBC/UKQCD Collaborations have made significant progress using the direct method, with results at nearly the physical quark masses and physical kinematics for the $\Delta I=3 / 2$ decay channel [49]; this is discussed below.

The calculation of $K \rightarrow \pi \pi$ decays from RBC/UKQCD uses the direct Lellouch-Lüscher approach, and they have made significant progress, completing a calculation of matrix elements in the $\Delta I=3 / 2$ channel with around $20 \%$ errors [50]. This required a large box size (around $4.5 \mathrm{fm}$ ) and physical light quark masses. The lightest unitary pion mass is $180 \mathrm{MeV}$, and a lighter valence pion with mass around $140 \mathrm{MeV}$ is used for the central value. The main errors contributing to the $\mathrm{RBC} / \mathrm{UKQCD}$ calculation of $\operatorname{Re}\left(A_{2}\right)$ are given in Table 1. The largest error is the estimate of scaling violations due to the use of somewhat coarse lattices at a single lattice spacing and the fact that $K \rightarrow \pi \pi$ matrix elements scale as the lattice spacing cubed.

The calculation of the $\Delta I=1 / 2$ rule is more difficult for a number of reasons. One is the presence of power divergent contributions arising from mixing under renormalization with lower dimensional operators. This problem has been addressed by the use of chiral fermions, where the operator subtraction is straightforward $[51,52]$. Another problem is the presence of enhanced finite-volume effects that afflict the calculation when the light valence quark masses are not the same as in the sea $[53,54]$. This was an especially serious problem for quenched attempts to calculate $\Delta I=1 / 2$ kaon matrix elements [55], but is under control when sea quarks of the correct masses are included in the calculation. Another difficulty comes from the fact that the physical amplitude is not the ground state when using the Lellouch-Luscher approach; this can be cured by implementing $G$-parity boundary conditions [45]. Yet another difficulty is the appearance of disconnected quark flow diagrams, leading to the need for very high statistics [56]. The contractions at the level of quark flow are shown in Fig. 5 for the $\Delta I=1 / 2$ channel. The red circle is the insertion of the four-quark operator. Additional diagrams with a quark current insertion are not shown, but 


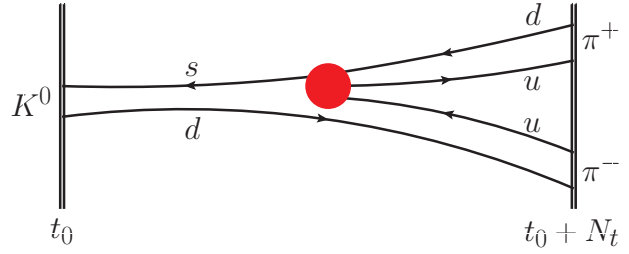

(a)

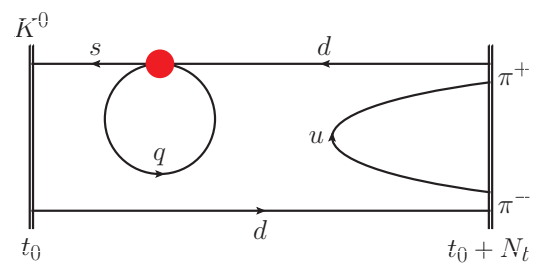

(c)

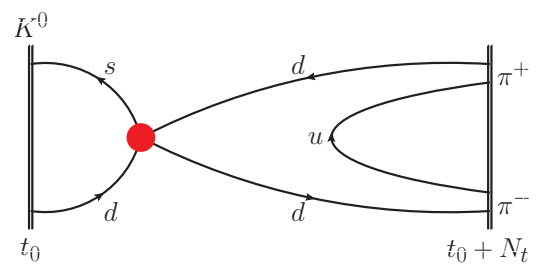

(b)

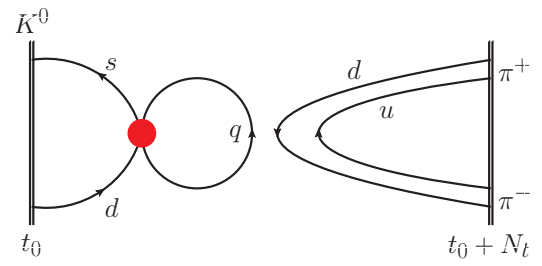

(d)

Figure 5: Quark flow diagrams for $K \rightarrow \pi \pi$ in the $\Delta I=1 / 2$ channel.

are needed to perform the power divergent operator subtraction. Figure 5(d) shows the disconnected diagram that is problematic due to the need for high statistics. This problem will likely be solved by more computing power and better inversion algorithms, both of which are now available, and a calculation is currently underway by RBC/UKQCD.

\section{Conclusion}

Lattice QCD is indispensable for the nonperturbative input needed to maximize constraints on new physics from kaon phenomenology. Quantities like kaon decay constants and semileptonic form-factors are now known at the sub-percent level. More difficult quantities like $K \rightarrow \pi \pi$ matrix elements in the $\Delta I=3 / 2$ channel are known at the 20\% [50]. The $\Delta I=1 / 2$ channel is more difficult but may be attainable in the next few years. Other difficult-to-compute quantities, such as the long distance contributions to kaon mixing [57] and rare kaon decays [58], may become available on a slightly longer time scale.

\section{References}

[1] S. Durr, arXiv:1305.5758 [hep-lat].

[2] J. Beringer et al. [Particle Data Group Collaboration], Phys. Rev. D 86, 010001 (2012).

[3] C. T. H. Davies, C. McNeile, K. Y. Wong, E. Follana, R. Horgan, K. Hornbostel, G. P. Lepage and J. Shigemitsu et al., Phys. Rev. Lett. 104, 132003 (2010) [arXiv:0910.3102 [hep-ph]].

[4] S. Durr, Z. Fodor, C. Hoelbling, S. D. Katz, S. Krieg, T. Kurth, L. Lellouch and T. Lippert et al., Phys. Lett. B 701, 265 (2011) [arXiv:1011.2403 [hep-lat]].

[5] C. Sturm, Y. Aoki, N. H. Christ, T. Izubuchi, C. T. C. Sachrajda and A. Soni, Phys. Rev. D 80, 014501 (2009) [arXiv:0901.2599 [hep-ph]].

[6] J. Laiho, E. Lunghi and R. S. Van de Water, Phys. Rev. D 81, 034503 (2010) [arXiv:0910.2928 [hep-ph]]. 
[7] A. Bazavov et al. [MILC Collaboration], PoS LAT 2009, 079 (2009) [arXiv:0910.3618 [hep-lat]].

[8] T. Blum, R. Zhou, T. Doi, M. Hayakawa, T. Izubuchi, S. Uno and N. Yamada, Phys. Rev. D 82, 094508 (2010) [arXiv:1006.1311 [hep-lat]].

[9] Y. Aoki et al. [RBC and UKQCD Collaborations], Phys. Rev. D 83, 074508 (2011) [arXiv:1011.0892 [hep-lat]].

[10] J. Laiho and R. S. Van de Water, PoS LATTICE 2011, 293 (2011) [arXiv:1112.4861 [hep-lat]].

[11] C. Kelly, PoS LATTICE 2011, 285 (2011) [arXiv:1201.0706 [hep-lat]].

[12] G. Colangelo, S. Durr, A. Juttner, L. Lellouch, H. Leutwyler, V. Lubicz, S. Necco and C. T. Sachrajda et al., Eur. Phys. J. C 71, 1695 (2011) [arXiv:1011.4408 [hep-lat]].

[13] W. J. Marciano, Phys. Rev. Lett. 93, 231803 (2004) [hep-ph/0402299].

[14] S. Durr, Z. Fodor, C. Hoelbling, S. D. Katz, S. Krieg, T. Kurth, L. Lellouch and T. Lippert et al., Phys. Rev. D 81, 054507 (2010) [arXiv:1001.4692 [hep-lat]].

[15] E. Follana et al. [HPQCD and UKQCD Collaborations], Phys. Rev. Lett. 100, 062002 (2008) [arXiv:0706.1726 [hep-lat]].

[16] A. Bazavov et al. [MILC Collaboration], PoS LATTICE 2010, 074 (2010) [arXiv:1012.0868 [hep-lat]].

[17] Y. Aoki et al. [RBC and UKQCD Collaborations], Phys. Rev. D 83, 074508 (2011) [arXiv:1011.0892 [hep-lat]].

[18] A. Bazavov, C. Bernard, C. DeTar, J. Foley, W. Freeman, S. Gottlieb, U. M. Heller and J. E. Hetrick et al., arXiv:1301.5855 [hep-ph].

[19] R. J. Dowdall, C. T. H. Davies, G. P. Lepage and C. McNeile, arXiv:1303.1670 [hep-lat].

[20] V. Lubicz, arXiv:1309.2530 [hep-lat].

[21] M. Antonelli, V. Cirigliano, G. Isidori, F. Mescia, M. Moulson, H. Neufeld, E. Passemar, M. Palutan et al., Eur. Phys. J. C69, 399-424 (2010). [arXiv:1005.2323 [hep-ph]].

[22] J. Gasser, H. Leutwyler, Nucl. Phys. B250, 465 (1985).

[23] M. Ademollo, R. Gatto, Phys. Rev. Lett. 13, 264-265 (1964).

[24] H. Leutwyler, M. Roos, Z. Phys. C25, 91 (1984).

[25] V. Lubicz et al. [ETM Collaboration], Phys. Rev. D 80, 111502 (2009) [arXiv:0906.4728 [hep-lat]].

[26] P. A. Boyle et al. [RBC-UKQCD Collaboration], Eur. Phys. J. C 69, 159 (2010) [arXiv:1004.0886 [hep-lat]].

[27] A. Bazavov, C. Bernard, C. M. Bouchard, C. DeTar, D. Du, A. X. El-Khadra, J. Foley and E. D. Freeland et al., arXiv:1212.4993 [hep-lat].

[28] T. Kaneko et al. [JLQCD Collaboration], PoS LATTICE 2012, 111 (2012) [arXiv:1211.6180 [hep-lat]].

[29] P. A. Boyle, J. M. Flynn, A. Juttner, C. Sachrajda, K. Sivalingam and J. M. Zanotti, PoS LATTICE 2012, 112 (2012) [arXiv:1212.3188 [hep-lat]].

[30] E. Gamiz, J. A. Bailey, A. Bazavov, C. Bernard, C. Bouchard, C. DeTar, D. Du and A. X. El-Khadra et al., PoS LATTICE 2012, 113 (2012) [arXiv:1211.0751 [hep-lat]]. 
[31] A. J. Buras and D. Guadagnoli, Phys. Rev. D 78, 033005 (2008).

[32] S. Durr, Z. Fodor, C. Hoelbling, S. D. Katz, S. Krieg, T. Kurth, L. Lellouch and T. Lippert et al., Phys. Lett. B 705, 477 (2011) [arXiv:1106.3230 [hep-lat]].

[33] E. Gamiz et al. [HPQCD and UKQCD Collaborations], Phys. Rev. D 73, 114502 (2006) [hep-lat/0603023].

[34] T. Bae, Y. -C. Jang, C. Jung, H. -J. Kim, J. Kim, J. Kim, K. Kim and S. Kim et al., Phys. Rev. Lett. 109, 041601 (2012) [arXiv:1111.5698 [hep-lat]].

[35] M. Antonelli, et al., Phys. Rept. 494, 197-414 (2010).

[36] L. Maiani and M. Testa, Phys. Lett. B 245, 585 (1990).

[37] C. W. Bernard, T. Draper, A. Soni, H. D. Politzer, M. B. Wise, Phys. Rev. D32, 2343-2347 (1985).

[38] P. Boucaud et al. [ The SPQ(CD)R Collaboration ], Nucl. Phys. Proc. Suppl. 106, 329-331 (2002). [hep-lat/0110206].

[39] J. Laiho, A. Soni, Phys. Rev. D65, 114020 (2002). [hep-ph/0203106].

[40] C. J. D. Lin, G. Martinelli, E. Pallante, C. T. Sachrajda, G. Villadoro, Nucl. Phys. B650, 301-355 (2003). [hep-lat/0208007].

[41] J. Laiho and A. Soni, Phys. Rev. D 71, 014021 (2005). [hep-lat/0306035].

[42] S. Li, N. H. Christ, PoS LATTICE2008, 272 (2008). [arXiv:0812.1368 [hep-lat]].

[43] J. Laiho, R. S. Van de Water, PoS LATTICE2010, 312 (2010). [arXiv:1011.4524 [hep-lat]].

[44] L. Lellouch and M. Lüscher, Commun. Math. Phys. 219, 31 (2001).

[45] C. -h. Kim, N. H. Christ, Nucl. Phys. Proc. Suppl. 119, 365-367 (2003). [hep-lat/0210003].

[46] N. H. Christ, C. Kim, T. Yamazaki, Phys. Rev. D72, 114506 (2005). [hep-lat/0507009].

[47] C. Kim, C. T. Sachrajda, S. R. Sharpe, PoS LAT2005, 359 (2006). [hep-lat/0510022].

[48] N. H. Christ [ RBC and UKQCD Collaborations ], PoS KAON09, 027 (2009). [arXiv:0912.2917 [hep-lat]].

[49] T. Blum, P. A. Boyle, N. H. Christ, N. Garron, E. Goode, T. Izubuchi, C. Jung and C. Kelly et al., Phys. Rev. Lett. 108, 141601 (2012) [arXiv:1111.1699 [hep-lat]].

[50] T. Blum, P. A. Boyle, N. H. Christ, N. Garron, E. Goode, T. Izubuchi, C. Jung and C. Kelly et al., Phys. Rev. D 86, 074513 (2012) [arXiv:1206.5142 [hep-lat]].

[51] J. I. Noaki et al. [ CP-PACS Collaboration ], Phys. Rev. D68, 014501 (2003). [hep-lat/0108013].

[52] T. Blum et al. [ RBC Collaboration ], Phys. Rev. D68, 114506 (2003). [hep-lat/0110075].

[53] G. Colangelo, E. Pallante, Nucl. Phys. B520, 433-468 (1998). [hep-lat/9708005].

[54] C. J. D. Lin, G. Martinelli, E. Pallante, C. T. Sachrajda, G. Villadoro, Phys. Lett. B581, 207-217 (2004). [hep-lat/0308014].

[55] C. J. D. Lin, G. Martinelli, E. Pallante, C. T. Sachrajda, G. Villadoro, Nucl. Phys. Proc. Suppl. 119, 371-373 (2003). [hep-lat/0209107].

[56] P. A. Boyle et al. [RBC and UKQCD Collaborations], arXiv:1212.1474 [hep-lat].

[57] N. H. Christ, T. Izubuchi, C. T. Sachrajda, A. Soni and J. Yu, arXiv:1212.5931 [hep-lat].

[58] C. T. Sachrajda, PoS KAON13, 019 (2013). 